\title{
Determinación de la capacidad mecánica de las bielas de los vehículos Kia Rio, Corsa Evolution y Volkswagen Gol mediante FEM y ensayos experimentales
}

\section{Determination of the mechanical capacity of the cranks of the Kia Rio, Corsa Evolution and Volkswagen Gol vehicles through FEM and experimental tests}

\author{
Rommel Paúl Imbaquingo Navarrete \\ Jorge Luis Melo Obando \\ Carlos Nolasco Mafla Yépez \\ Ignacio Bayardo Benavides Cevallos \\ Universidad Técnica del Norte, Ecuador \\ Autor por correspondencia: rpimbaquingo@utn.edu.ec (0983514647),jlmelo@utn.edu.ec \\ (0982441661), cnmafla@utn.edu.ec (0999674713), ibbenavides@utn.edu.ec (0997236253). \\ Fecha de recepción: 26 de febrero de 2018 - Fecha de aceptación: 15 de agosto de 2018
}

Resumen: Este artículo se basa en la determinación de la capacidad mecánica que soportan las bielas de los vehículos Kia Rio, Corsa Evolution y Volkswagen Gol, en base a un análisis estático por compresión y pandeo en condiciones de columna, determinando si existe o no sobredimensionamiento de estas. El estudio se desarrolla en fases: Análisis químico realizado por un espectrómetro de chispa, ensayos de dureza del material y análisis micrográfico basado en normas ASTM, determinando el material específico de cada biela. Cálculo analítico determinando la presión y fuerza de explosión en el motor. Análisis de las bielas en condiciones de columna logrando obtener valores críticos a los cuales las bielas empezarán a pandearse, estos valores son analizados mediante FEM pudiendo determinar los factores de seguridad para cada caso. Modelado y Análisis FEM a compresión de cada Biela con incremento de carga hasta obtener los valores de esfuerzo, desplazamiento y factor de seguridad últimos. Realización de ensayos no destructivos por tintas penetrantes y partículas magnéticas basado en normas ASTM determinando si existe o no posibles fisuras o porosidades que influencien en los resultados. Ensayo experimental a compresión en la máquina de ensayos universales obteniendo resultados que serán comparados con los obtenidos mediante FEM. Comprobación de sobredimensionamiento en las bielas mediante comparación de esfuerzos obtenido del ensayo experimental y mediante FEM considerando la fuerza en el ciclo de expansión. Determinación de un modelo matemático usando regresiones lineales del cual obtendremos el esfuerzo en función de la fuerza de compresión que ingresemos.

Palabras Claves: biela; FEM; análisis micrográfico; tintas penetrantes; ASTM

\begin{abstract}
This article is based on the determination of the mechanical capacity of the Connecting rod of the Kia Rio, Corsa Evolution and Volkswagen Gol vehicles, based on a static analysis by compression and buckling under column conditions, determining whether or not there is overdimensioning of the same. The study is developed in phases: Chemical analysis performed by a
\end{abstract}


spark spectrometer, material hardness tests and micrographic analysis based on ASTM standards, determining the specific material of each connecting rod. Analytical calculation determining the pressure and explosion force in the engine. Analysis of the connecting rods in column conditions, obtaining critical values to which the connecting rods will begin to buckle, these values are analyzed by means of EMF, being able to determine the safety factors for each case. Modeling and FEM compression analysis of each connecting rod with load increase until the last effort, displacement and safety factor values are obtained. Performing non-destructive tests by penetrating inks and magnetic particles based on ASTM standards determining whether or not there are possible cracks or porosities that influence the results. Experimental compression test in the universal testing machine obtaining results that will be compared with those obtained by FEM. Checking oversize in the connecting rods by comparing the forces obtained from the experimental test and using FEM considering the force in the expansion cycle. Determination of a mathematical model using linear regressions from which we will obtain the effort as a function of the compression force that we enter.

Key words: connecting rod; FEM; micrographic analysis; penetrating inks; ASTM

\section{Introducción}

Strozzi, Baldini, Giacopini, Bertocchi \& S. Mantovani (2015) en cuanto a la definición de la biela expresan que es uno de los componentes más importantes empleados en los motores de combustión interna. Sus extremidades están configuradas como ojos, denominados como extremo de vástago (superior) y vástago grande (inferior), conectados por un vástago en forma de viga. El extremo pequeño de la biela está unido al pistón por medio del pasado, mientras que el extremo grande está montado en el cigüieñal

Además, Strozzi et al. (2015) refieren que la función de la biela es trasladar el movimiento transversal alterno del pistón a movimiento de rotación del cigüeñal y como parte del motor, la biela se somete a cargas de fatiga de alto ciclo. Deben tenerse en cuenta las contribuciones debidas tanto a las fuerzas de compresión como a las fuerzas de inercia. La biela tiene que ser lo suficientemente fuerte como para soportar la carga externa, lo suficientemente rígida para permitir un acoplamiento correcto con el pasador y la manivela y, al mismo tiempo, tiene que ser lo suficientemente ligera como para minimizar las fuerzas inerciales derivadas de su movimiento. En particular, una cierta porción de la biela puede considerarse como una masa alterna, afectando así directamente al valor máximo de su fuerza alterna efectiva.

Como consecuencia, se debe prestar especial atención al proceso de diseño de la biela, ambos métodos tanto analítico como numérico se utilizan generalmente para la optimización de la biela.

Mohsin \& Haneef (2015) expresa que si bien la biela de un motor de combustión interna está expuesta a grandes cargas cíclicas de trabajo del orden de $10^{5}$ a $10^{9}$ ciclos debido a las cargas generadas en el tiempo de explosión es importante la durabilidad y fiabilidad de este componente para el excelente desempeño del automotor.

Para el caso en estudio, existe el análisis desarrollado con el fin de analizar la fractura ocurrida en la biela de un compresor de refrigeración, investigación desarrollada por Ernesto 
Germán Porras, Sara Rodríguez Pulecio \& John Jairo Coronado (2010) con el objetivo de determinar las razones por las que falló la biela, en tal estudio detallan diversos factores que dieron origen a este hecho mencionando que los ciclos de carga de compresión, aumento de coeficiente de rozamiento, la pérdida de lubricación y la presencia de porosidades aumentaron los esfuerzos en la biela conllevando a una lubricación límite, al incremento del daño superficial y desgaste por deslizamiento que a su vez éste incremento fue influenciado por la porosidad y las asperezas, la fuerza tangencial fue muy alta que rompió la microsoldadura conduciendo a valores inaceptables de fricción, desgaste y aumento de temperatura provocando esfuerzos de flexión en la biela los cuales produjeron la fractura de forma dúctil luego chocó la biela contra el bloque del compresor y se fracturó de forma frágil debido al impacto, todo esto validado con métodos analíticos en el cálculo de esfuerzos, inercia, etc. y experimentales con análisis micrográficos, fractografías y microscópicos.

En un estudio realizado en Italia por Mattia Pujatti, Mitja Suhadolc \& Daniel Piculin (2014) enfocado en analizar el desgaste originado en las bielas de los motores de gran tamaño pudieron determinar que existían varios factores que originaron la causa entre ellos el número extremadamente alto de ciclos de trabajo por encima de 108, presión radial demasiado baja del cojinete produciendo juego con la biela de esta manera se originó puntos de adhesión, grietas iniciadas por fricción en la superficie de la carcasa, el crecimiento de fisuras puramente por fatiga fue entonces posible y condujo al fracaso de la biela, métodos analíticos como la ecuación de El Haddad Topper Smith, ensayos experimentales como ensayos no destructivos por partículas magnéticas sirvieron de ayuda para determinar que por la presión radial del cojinete que no era suficiente para evitar el juego, las grietas se iniciaron y crecieron bajo tensiones oscilantes, la altura de aplastamiento del cojinete que brinde una correcta presión radial y así disminuir el deslizamiento entre el casquillo de cojinete y la carcasa no era la correcta, además se debía mejorar la resistencia al deslizamiento y reducir la fricción.

En la India, Saharash Khare, O.P. Singh, K. Bapanna Dora \& C. Sasun (2012), en su investigación debido al problema por alto ruido y vibraciones en un motor por rotura de una biela a temprano tiempo de vida útil, se determinó un factor preponderante que conllevó a los síntomas antes mencionado, siendo éste el desgaste en las superficies de contacto de los pasadores, los cojinetes y las superficies de los extremos grandes de la biela el cual mediante análisis de las diversas partes que conforman el motor, análisis por elementos finitos, ensayos en dinamómetro y el método de ensayo controlado por matriz de cuatro cuadrantes cuyo método consiste en correlacionar básicamente el MTTF (tiempo medio al fallo) en pruebas de campo con el MTTF en laboratorio se pudo determinar que existía grietas superficiales en el pasador de manivela, los rodamientos de rodillos y las superficies de los extremos grandes de las bielas (bulón del cigüeñal), además existía una alta presión interfacial y tensiones cerca de la unión de la banda y la brida de la biela y ante todo esto se modificó la biela mediante FEM mostrando una reducción significativa de la presión, lo que dio como resultado una mejora significativa de la durabilidad en la prueba de laboratorio.

Existe también estudios en China referente al daño de las bielas, investigación desarrollada por Bai-yan He, Guang-da Shi, Ji-bing Sun, Si-zhuan Chen \& Rui Nie (2013), con la finalidad de analizar la falla en una biela de un grupo electrógeno diésel determinaron múltiples factores que originaron el fallo de la biela entre ellos son los valores relativamente 
bajos en cuanto a las propiedades mecánicas del material como la resistencia a la tracción, el límite elástico del material, la tensión de los pernos y las superficies coincidentes dentadas eran relativamente altas, determinando que la tensión máxima de la biela se produjo en la superficie de acoplamiento cerca del orificio del perno de sujeción, además se realizó el examen metalográfico y fractografías indicando que la fatiga es el principal mecanismo de las grietas y el fallo de la biela y que el alto esfuerzo de montaje del perno junto con la tensión del ciclo durante las operaciones del motor podría causar la generación y extensión de las grietas, en cuanto al material todos estos resultados fueron logrados gracias a ensayos de dureza, composición química y análisis de elementos finitos no lineales en el esfuerzo de montaje de los pernos.

\section{Metodología}

La determinación del material que conforman las bielas se realizó de acuerdo con los siguientes métodos:

\section{Análisis químico}

Para determinar el porcentaje de cada material que conforman las bielas se realizó la cuantificación de los metales presentes en la muestra empleando el Espectrómetro de Chispa marca BRUKER modelo Q4TASMAN.

\section{Análisis de dureza}

Se determinó la dureza de cada material que conforman las bielas en tres puntos distintos de cada probeta cuyo promedio es el valor de la dureza del material. La escala usada fue Rockwell B (HRb).

\section{Análisis metalográfico}

El análisis metalográfico inicia con un proceso de corte transversal del cuerpo de biela con el fin de obtener las probetas, seguidamente tenemos el pulido de estas hasta obtener un acabado tipo espejo para proceder al ataque químico con nital al 3\%. Este análisis se basa en las normas ASTM E3 y ASTM E112.

\section{Comprobación de sobredimensionamiento}

La comprobación del sobredimensionamiento se basa en una comparación del esfuerzo generado mediante FEM considerando la fuerza en el ciclo de explosión y el esfuerzo generado por la fuerza máxima encontrada en el ensayo experimental sobre el área de la sección transversal.

Para este desarrollo se lleva a cabo el cálculo de la presión y la fuerza de explosión en el ciclo de expansión, análisis de la biela en condiciones de columna, ensayo experimental en la máquina de ensayos universales, previa verificación de posibles fisuras y porosidades mediante análisis no destructivos. 
Cálculo de la presión y fuerza de explosión en el ciclo de expansión

Mediante el libro "Motores del Automóvil" cuyos autores son Jóvaj \& Trusov (1982) se obtuvieron los valores de presión y fuerza en el ciclo de expansión, se ha desarrollado una serie de cálculos los cuales resultan ser una secuencia desde el ciclo de admisión y compresión hasta finalmente llegar al ciclo de explosión en cuanto a las presiones y temperaturas de cada ciclo.

\section{Análisis de la biela en condiciones de columna}

En base a los libros titulados "Diseño de Elementos de Máquinas" cuyo autor es Mott (1992), "Diseño en Ingeniería Mecánica" cuyos autores son Shigley \& Milshchke (2005) y "Diseño de máquinas" de Norton (1999) se procede a realizar el análisis en condiciones de columna determinando previamente si las bielas trabajan como columnas cortas o largas y aplicar la ecuación respectiva para cada tipo, es decir, ecuación de J.B. Johnson o Euler respectivamente, adicional se necesita previamente haber determinado el material que conforman las bielas para así poder obtener datos en cuanto a su módulo de elasticidad, la resistencia última a la tensión, el área de la sección transversal del cuerpo de la biela, la longitud real de la columna y la constante en base a la fijación de los extremos, datos que nos llevarán a la realización de cálculos en base al momento de inercia, Radio de giro, Longitud efectiva de la columna y la constante de la columna, para poder definir si la biela actúa como columna corta o larga y usar la ecuación de Johnson o Euler respectivamente dependiendo el caso, en base a todos los cálculos se pudo determinar que todas actúan como columnas cortas dando los valores críticos para cada caso y que al analizarlas por pandeo mediante FEM obtendremos el factor de seguridad referente a la carga crítica obtenida.

\section{Ensayo experimental a compresión}

Para la ejecución del ensayo experimental se realizó el análisis no destructivo a las tres bielas tanto por tintas penetrantes como por partículas magnéticas donde no se detectaron fisuras ni porosidades que resulten como concentradores de tensión y así disminuya la capacidad de carga.

El ensayo no destructivo se realizó utilizando el procedimiento de tintas penetrantes basado en la norma ASTM E 165 y por partículas magnéticas basado en la norma ASTM E 709. El estudio experimental se ha realizado en la máquina de ensayos universales TIME cuyas lecturas de la gráfica y deformación se realizaron desde el respectivo software de la máquina.

La carga aplicada está dada en kilo newtons $(\mathrm{KN})$, a una velocidad de $1 \mathrm{~mm} / \mathrm{min}$ y un modo de visualización Desplazamiento vs Carga. Para este ensayo se realizó la simulación del bulón y del muñón del cigüeñal con cilindros cuyo material fue acero de transmisión.

\section{Ensayo a compresión por método de elementos finitos FEM}


Se desarrolló el análisis por elementos finitos en el software de modelado y simulación SolidWorks en el cual se ha procedido a realizar un estudio a cada biela tomando en cuenta un análisis por compresión y por fallo por condiciones de columna o también denominado Pandeo.

Al tener completamente modeladas las bielas y con ayuda de los análisis para la determinación del material se asigna el material para cada caso además de su respectivo ensamble y sus respectivos aditamentos que simulan al bulón y al muñón del cigüeñal como se realizó en el ensayo experimental.

Se procede a realizar en análisis a compresión y por condiciones de columna mediante incrementos de carga de $20 \mathrm{KN}$ hasta el valor máximo obtenido en el análisis experimental verificando la falla de las bielas y validando entre sí los datos del estudio práctico en la máquina de ensayos universales. Los resultados se obtuvieron en base al esfuerzo, desplazamiento y factor de seguridad.

\section{Modelo matemático}

Mediante la utilización de regresiones lineales se logró determinar un modelo matemático el cual nos brindará los datos del esfuerzo dependiendo la variable fuerza que ingresemos.

\section{Resultados}

En la tabla 1 se muestran los resultados del ensayo por análisis químico de los materiales que conforman cada biela.

Tabla 1. Resultados del Análisis Químico

\begin{tabular}{cccc}
\hline METALES & VOLKSWAGEN GOL (\%) & KIA RIO (\%) & CHEVROLET CORSA (\%) \\
\hline Carbono (C) & 0.393 & 0.616 & 2.992 \\
Silicio (Si) & 0.217 & 0.168 & 2.087 \\
Manganeso (Mn) & 0.737 & 0.858 & 0.183 \\
Cromo (Cr) & 0.105 & 0.094 & 0.036 \\
Níquel (Ni) & 0.043 & 0.038 & 0.019 \\
Fósforo (P) & $<0.005$ & $<0.005$ & 0.004 \\
Azufre (S) & $<0.150$ & $<0.150$ & $<0.001$ \\
Cobre (Cu) & 0.129 & 0.206 & 0.415 \\
Aluminio (Al) & 0.035 & $<0.005$ & $<0.001$ \\
Molibdeno (Mo) & 0.012 & 0.015 & 0.002 \\
Titanio (Ti) & 0.003 & $<0.001$ & 0.020 \\
Vanadio (V) & $<0.005$ & $<0.005$ & 0.004 \\
Boro (B) & $<0.001$ & $<0.001$ & $<0.0002$ \\
\hline
\end{tabular}




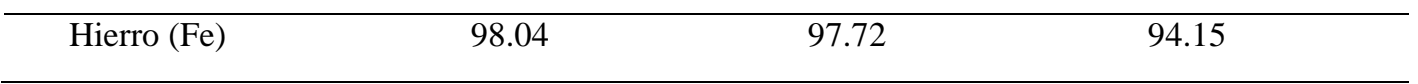

En la tabla 2 se evidencia los resultados de la dureza en la escala Rockwell B ensayadas en el material de cada biela.

Tabla 2. Resultados ensayo de dureza

\begin{tabular}{ll}
\hline Vehículo & Dureza (HRB) \\
\hline Volkswagen Gol & 98,33 \\
& \\
Kía Río & 95,66 \\
Chevrolet Corsa & 99,83 \\
\hline
\end{tabular}

Después del fotografiado microscópico se obtiene las micrografías del material que conforman las bielas cuyos gráficos se muestran en la tabla 2 .

De acuerdo con el análisis químico, de dureza, de micrografía y en comparación con el libro Metals Handbook ninth edition (s.f.) correspondiente a METALLOGRAPHY and MICROSTRUCTURES ASM podemos determinar que el material de la biela del vehículo Chevrolet Corsa corresponde a una Fundición Nodular matriz Perlítica, del vehículo Kía Río corresponde a un acero AISI 1060 Matriz perlítica y ferrítica y del vehículo Volkswagen Gol corresponde a un acero AISI 4140. Matriz Martensítica.

Tabla 3. Análisis Metalográfico

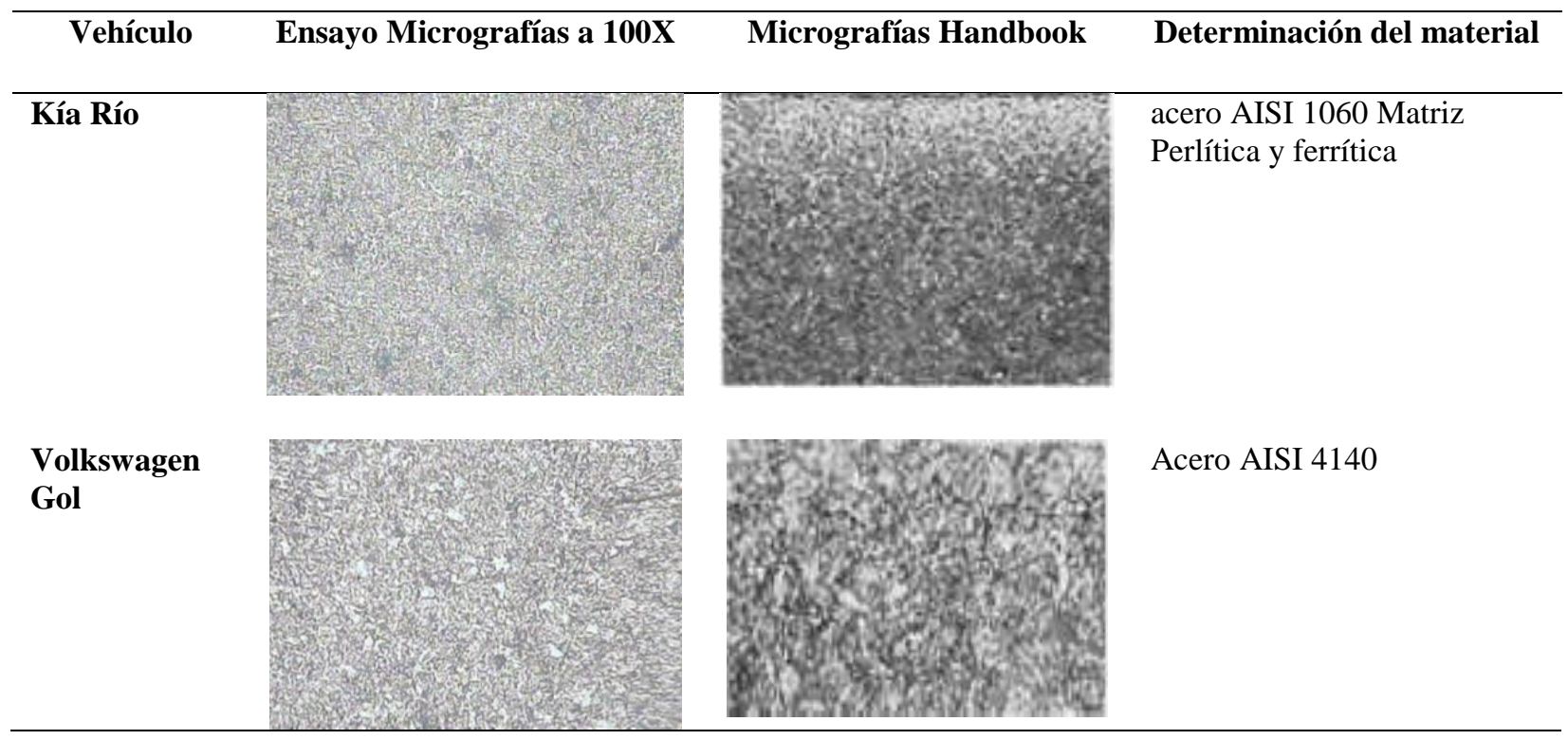


Chevrolet Corsa
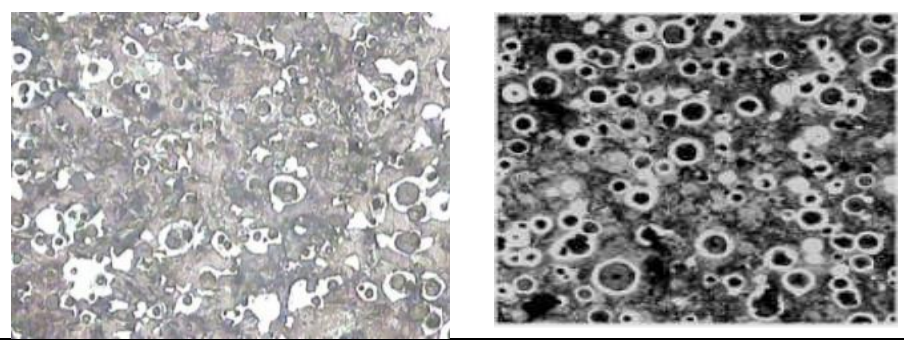

Fundición Nodular matriz

Perlítica

Para la comprobación de un posible sobredimensionamiento como se dijo anteriormente se procede a una serie de cálculos como son: Cálculo de la presión y fuerza de explosión en el ciclo de expansión, análisis de la biela en condiciones de columna, ensayo experimental a compresión.

Para el primer caso se obtuvo la presión y fuerza de explosión en el ciclo de expansión basada en el libro de M.S. Jovaj.

Tabla 4. Valores de presión y fuerza en el ciclo de explosión

\begin{tabular}{ccc}
\hline Automóvil & Pz (MPa) & Fe(N) \\
\hline Kía Río & 4,83 & 21623,4 \\
Volkswagen Gol & 3,77 & 19640,45 \\
Chevrolet Corsa & 4,59 & 23390,83 \\
\hline
\end{tabular}

Pz: Presión en el ciclo de explosión

Fe: Fuerza de explosión

Para las condiciones de columna previamente se determina si las bielas trabajan como columna corta o larga para utilizar las debidas ecuaciones tanto de J.B. Johnson o Euler respectivamente. Se determinó que las bielas trabajan como columna corta tras una serie de cálculos que el análisis exige.

$$
\begin{aligned}
& \text { Ecuación de J.B.Johnson } \\
& P_{C r}=A S_{y}\left[1-\frac{S_{y}\left(\frac{K L^{2}}{r}\right)}{4 \pi^{2} \epsilon}\right]
\end{aligned}
$$

Siendo:

$P_{C r}=$ carga crítica $(N)$

$A=$ área de la sección transversal $\left(\mathrm{m}^{2}\right)$

$S_{y}=$ resistencia última a la tensión $\left(M P_{a}\right)$ 


$$
\begin{aligned}
& k=\text { constante de columna }(1) \\
& L=\text { longitude real de la columna }(m) \\
& r=\text { radio de giro de la columna }(m) \\
& \epsilon=\text { módulo de elasticidad }\left(G P_{a}\right)
\end{aligned}
$$

En la tabla 5 se muestran los resultados de las bielas al ser analizadas en condiciones de columna, pudiendo obtenerse la carga crítica a la cual la biela empezará a pandearse juntamente con el factor de seguridad validando esa carga crítica para cada biela al obtenerlo mediante análisis FEM.

Tabla 5. Resultados análisis en condiciones de columna

\begin{tabular}{ccc}
\hline Automóvil & PCr (N) & Factor de Seguridad \\
\hline Kía Río & 57268,01 & 1,07 \\
Volkswagen Gol & 79288,92 & 1,1 \\
Chevrolet Corsa & 47285,62 & 1,01 \\
\hline
\end{tabular}

Antes de realizar el ensayo a compresión en la máquina de ensayos universales se efectúa

\begin{tabular}{|c|c|c|}
\hline $\begin{array}{c}\text { Ensayo no } \\
\text { destructivo }\end{array}$ & Figura & Conclusión \\
\hline $\begin{array}{c}\text { Tintas } \\
\text { penetrantes }\end{array}$ & & $\begin{array}{c}\text { no se detectaron fisuras } \\
\text { superficiales ni } \\
\text { porosidades. }\end{array}$ \\
\hline $\begin{array}{l}\text { Partículas } \\
\text { Magnéticas }\end{array}$ & & $\begin{array}{l}\text { no existen fisuras, } \\
\text { porosidades o } \\
\text { discontinuidades }\end{array}$ \\
\hline
\end{tabular}
en este análisis de ensayos no destructivos por tintas penetrantes y partículas magnéticas. Los resultados del ensayo se muestran a continuación:

Tabla 6. Resultados ensayos no destructivos 
Las pruebas se realizaron con incremento de carga cada $20 \mathrm{KN}$ obteniendo una carga máxima de 116, $22 \mathrm{KN}$ para la biela del vehículo Kía Río, para la biela del vehículo Volkswagen Gol se obtuvo una carga máxima de 111,02KN y del vehículo Chevrolet Corsa una carga máxima de $98,5 \mathrm{KN}$. El esfuerzo para cada uno se obtuvo dividiendo la fuerza máxima determinada mediante el ensayo experimental sobre el área de la sección transversal.

Resultados del ensayo experimental a compresión:

Tabla 7. Resultados ensayo Experimental

\begin{tabular}{cccc}
\hline Automóvil & Carga $(\mathbf{N})$ & Área $\left(\mathbf{m}^{\mathbf{2}}\right)$ & Esfuerzo Calculado \\
\hline Kía Río & 116220 & 0,000151 & 769,67 \\
Volkswagen Gol & 111024 & 0,000146 & 760,43 \\
Chevrolet Corsa & 98500 & 0,000192 & 513,02 \\
\hline
\end{tabular}

Finalmente se determina si las bielas de los vehículos anteriormente nombrados presentan un sobredimensionamiento o no mediante la comparación del esfuerzo generado mediante FEM considerando la fuerza en el ciclo de explosión y el esfuerzo generado por la fuerza máxima encontrada en el ensayo experimental sobre el área de la sección transversal.

El esfuerzo FEM se ha obtenido del software de simulación el cual fue calculado en base a la fuerza encontrada en el ciclo de explosión y para el cálculo del esfuerzo en base a la fuerza máxima experimental se ha usado la ecuación (2), la cual involucra la fuerza máxima que se obtuvo de la máquina de ensayos universales y el área de la sección transversal para cada marca de vehículo:

$$
\begin{aligned}
& \qquad \sigma c_{F}=\frac{F}{A} \\
& \sigma c_{F}=\text { esfuerzo a compresión }\left(M P_{a}\right) \\
& F=\text { fuerza máxima experimental }(N) \\
& A=\text { área de la sección transversal }\left(\mathrm{m}^{2}\right)
\end{aligned}
$$

Tabla 8 Resultados de sobredimensionamiento

$\begin{array}{ccc}\text { Vehículo } & \begin{array}{c}\text { Esfuerzo en base a la } \\ \text { fuerza máxima } \\ \text { experimental } \sigma c_{F}\end{array} & \begin{array}{c}\text { Esfuerzo FEM en } \\ \text { base a la fuerza } \\ \text { de explosión } \sigma c_{F e}\end{array}\end{array}$ Conclusión




\begin{tabular}{|c|c|c|c|}
\hline $\begin{array}{l}\text { Volkswagen } \\
\text { Gol }\end{array}$ & 760,4 & 130,66 & $\begin{array}{l}\text { Existe sobredimensionamiento ya que el } \\
\text { esfuerzo calculado en base a la fuerza del }\end{array}$ \\
\hline $\begin{array}{l}\text { Chevrolet } \\
\text { Corsa }\end{array}$ & 513,02 & 132,29 & $\begin{array}{l}\text { ensayo experimental es mucho mayor que el } \\
\text { generado dentro del ciclo de explosión. }\end{array}$ \\
\hline
\end{tabular}

Para la validación de los ensayos tanto experimental como FEM también se procedió a analizar las bielas en cuanto a su carga máxima que éstas pudieran soportar en el software SolidWorks, se comparó los esfuerzos obtenidos mediante FEM y mediante el ensayo experimental dando valores bastante aproximados y con un factor de seguridad menor o igual a uno indicando que en realizad es la carga máxima que pueden soportar dichas bielas, además de validar los resultados y la correcta realización de los ensayos. En la tabla 8 se muestran los resultados analizados mediante FEM considerando la fuerza máxima experimental.

Tabla 9. Resultados ensayo FEM a compresión.

\begin{tabular}{cccc}
\hline Automóvil & Fuerza Máxima & Esfuerzo (MPa) & Factor de Seguridad \\
\hline Kía Río & 116220 & 799,35 & 0,97 \\
Volkswagen Gol & 111024 & 738,62 & 1,03 \\
Chevrolet Corsa & 98500 & 557,08 & 0,95 \\
\hline
\end{tabular}

Basado en libro cuyos autores son Walpole \& Ronald E (1999) titulado "Probabilidad y estadística para ingenieros" y en el libro de Sheldon (2007) titulado "Introducción a la estadística", con el tema de regresiones lineales se logró determinar un modelo matemático el cual nos brindará los datos del esfuerzo dependiendo la variable fuerza que ingresemos.

Tabla 10. Modelo de Regresión Lineal

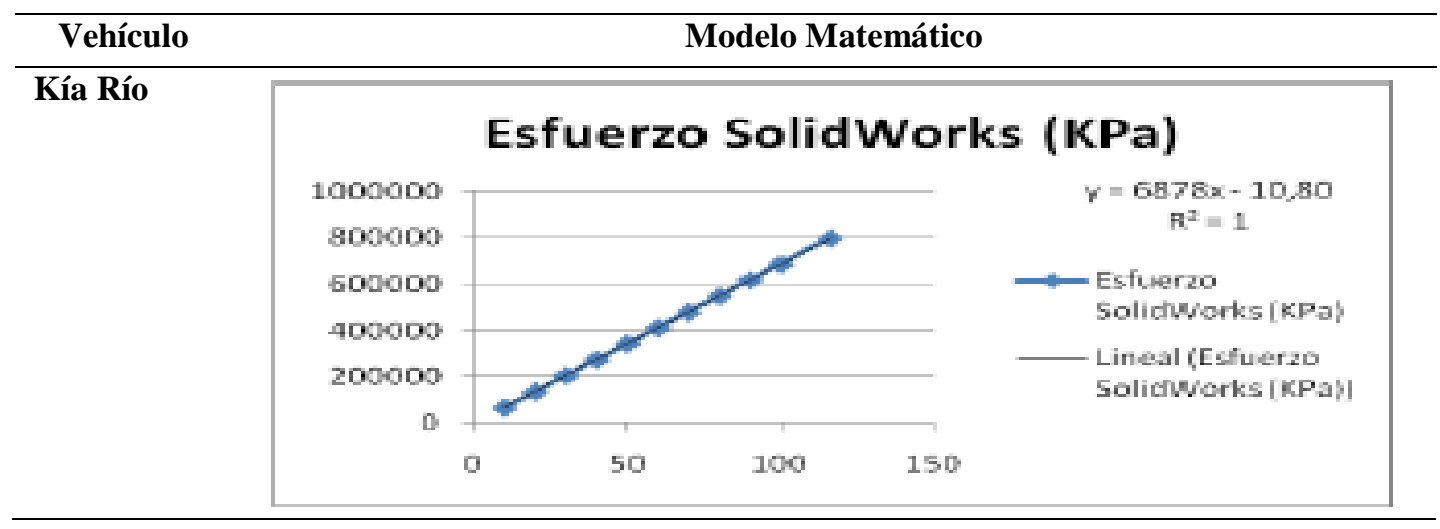




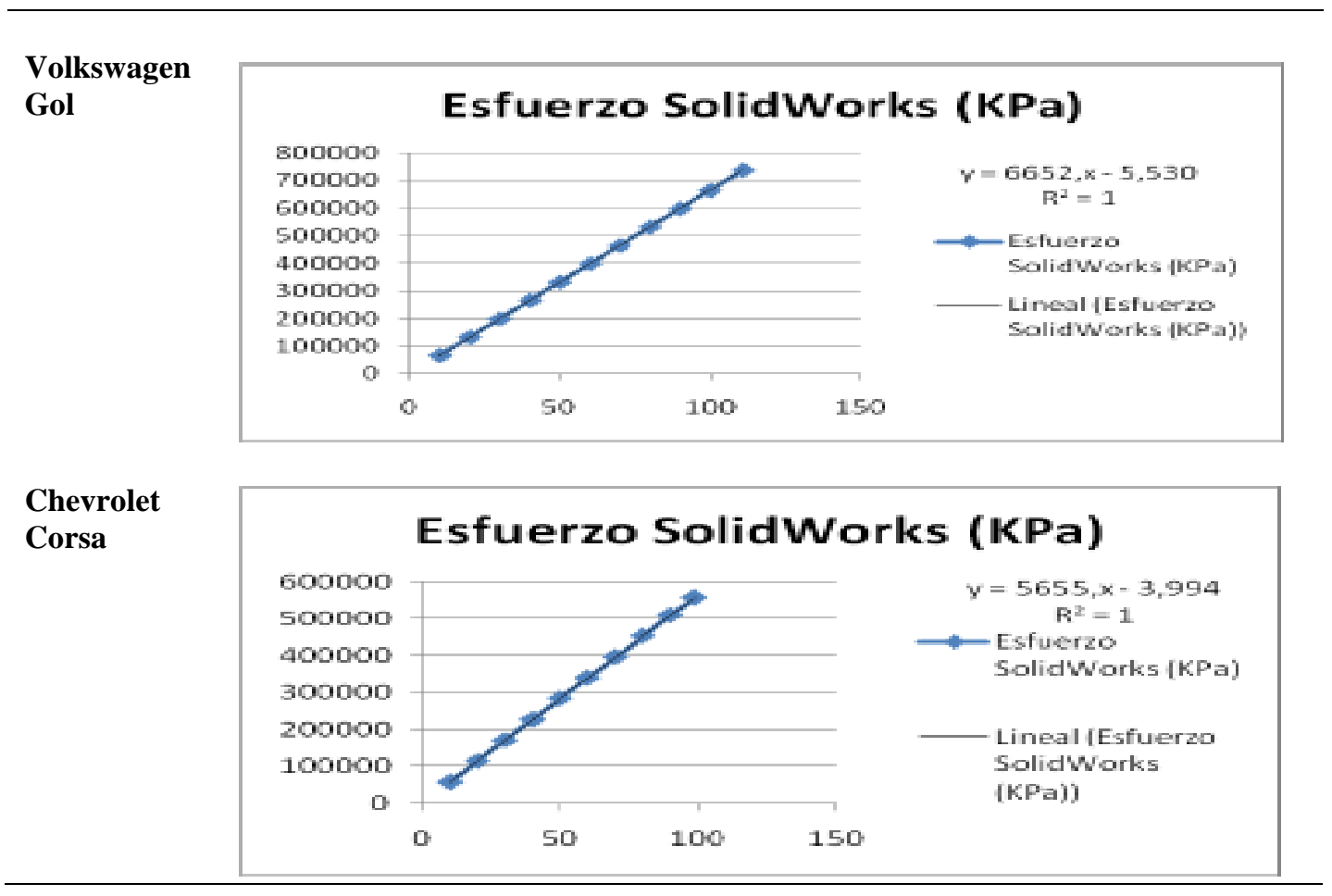

Discusión

En la presente investigación y en base a los resultados de las micrografía llama la atención que el elemento del Carbono es más abundante en la biela del vehículo Corsa Evolution comparado con los otros dos vehículos, primeramente corroborando la determinación del material Fundición Nodular Gris cuya principal característica es el alto contenido de carbono el cual proporciona mayor dureza pero mayor facilidad a la rotura, por eso es que en los ensayos experimentales ésta llego a romperse mientras que las bielas de los otros vehículos se doblaron pero no se partieron.

De los resultados en cuanto a determinar un posible sobredimensionamiento en las capacidades mecánicas de cada biela se puede observar que todas cumplen las exigencias de cada motor.

Tabla 11. Resultados del Sobredimensionamiento de las bielas de cada vehículo

\begin{tabular}{cccc}
\hline Vehículo & $\begin{array}{c}\text { Esfuerzo en base a la fuerza } \\
\text { máxima experimental } \boldsymbol{\sigma} \boldsymbol{c}_{\boldsymbol{F}} \\
\left(\boldsymbol{M P} \boldsymbol{P}_{\boldsymbol{a}}\right)\end{array}$ & $\begin{array}{c}\text { Esfuerzo FEM en base a la } \\
\text { fuerza de explosión } \boldsymbol{\sigma} \boldsymbol{c}_{\boldsymbol{F e}} \\
\left(\boldsymbol{M P} \boldsymbol{P}_{\boldsymbol{a}}\right)\end{array}$ & $\begin{array}{c}\text { Factor de } \\
\text { Seguridad }_{\boldsymbol{\sigma} \boldsymbol{c}_{\boldsymbol{F}} / \boldsymbol{\sigma} \boldsymbol{c}_{\boldsymbol{F} \boldsymbol{e}}}\end{array}$ \\
\hline $\begin{array}{c}\text { Kía Río } \\
\text { Volkswagen } \\
\text { Gol }\end{array}$ & 769,66 & 148,724 & \\
$\begin{array}{c}\text { Chevrolet } \\
\text { Corsa }\end{array}$ & 760,4 & 130,66 & 5.1 \\
\hline
\end{tabular}


Los esfuerzos obtenidos tanto del ensayo experimental y por elementos finitos son bastante próximos validando ambos métodos en cuanto a la obtención de las capacidades mecánicas.

Tabla 12. Comparación de esfuerzos obtenidos del ensayo experimental y por FEM.

\begin{tabular}{ccc}
\hline Automóvil & $\begin{array}{c}\text { Esfuerzo Ensayo experimental } \\
\text { (MPa) }\end{array}$ & $\begin{array}{c}\text { Esfuerzo por ensayo FEM } \\
\end{array}$ \\
& & (MPa) \\
\hline Kía Río & 769,67 & 799,35 \\
Volkswagen Gol & 760,43 & 738,62 \\
Chevrolet Corsa & 513,02 & 557,08 \\
& & \\
\hline
\end{tabular}

\section{Conclusiones}

Con ayuda del análisis químico y de la microestructura se determinó que la biela del Chevrolet Corsa correspondía a una fundición nodular Gris 80-55-06, mientras que en Volkswagen Gol y Kía Río eran aceros con bajo contenido de carbono, AISI 1060 y AISI 4140 respectivamente.

De los ensayos realizados se pudo determinar que la biela de Chevrolet Corsa por su alto contenido de carbono presentaba mayor dureza, pero a la vez mayor fragilidad a la rotura, lo que no sucedía con la biela del Volkswagen Gol y del Kía Río que por su bajo contenido de carbono no presentaban mucha fragilidad sino plasticidad.

Se determinó la máxima carga que pueden soportar las bielas al ser sometidas al esfuerzo de compresión, mediante la ayuda de ensayos experimentales y por elementos finitos.

El software nos proporciona un modelado de la biela, así como la facilidad para aplicarle el esfuerzo de compresión y un análisis por columna, obteniendo así diferentes valores de carga para posteriormente ser comparados con los obtenidos experimentalmente.

Determinamos que las bielas de cada vehículo se encuentran sobredimensionadas ya que la carga generada en el ciclo de explosión es menor que la carga crítica calculada como columna corta y menor que la fuerza máxima de compresión.

El sobredimensionamiento y la confiabilidad hace que las bielas sean difíciles de ser remplazadas en modificaciones que se realice dentro del motor, implicando aumento de presiones o cargas.

\section{Bibliografía}

Askeland, D. (2006). Ciencia e Ingeniería de los Materiales tercera edición. Thomson. 
ASM Metals Handbook vol 9. (s.f.). Metallography and Microstructures . ASM.

ASTM E 112. (n.d.). Determinación del tamaño de grano .

ASTM E 165. (n.d.). Ensayos no destructivos por tintas penetrantes .

ASTM E 3 . (n.d.). Preparación de muestras metalográficas .

ASTM E 709. (n.d.). Ensayos no destructivos por partículas magnéticas .

Baiyan , H. A., Guang-da, S. A., Ji-bing, S., Si-Zhuan, C., \& Nie , R. (2013). Crack analysis on the toothed mating surfaces of a diesel engine connecting rod . ELSEVIER , 443-450.

Jóvaj , M. S., \& Trúsov , V. I. (1982). Motores del Automovil . Moscú: MIR Moscú .

Khare, S., Singh , O. P., Bapanna k. , D., \& Sasun , C. (2012). Spalling investigation of connecting rod . ELSEVIER , 77-86.

Mohammed Mohsin , A. H., \& Mohamed , H. (2015). Analysis of Fatigue Stresses on Connecting Rod Subjected to Concentrated Loads At The Big End. ELSEVIER, 2094-2103.

Mott, R. (1992). Diseño de elementos de máquinas. . Hispanoamericana .

Norton , R. (1999). Diseño de Máquinas primera edición . México : PEARSON .

Porras , E. G., Rodríguez Pulecio, S., \& Coronado , J. J. (2010). Análisis de la fractura de una biela de compresor de refrigeración . Ingeniería e Investigación , 130-135.

Pujattia, M., Suhadolca, M., \& Piculina, D. (2014). Fretting-initiated fatigue in large bore engines connecting rods . ELSEVIER, 356-359.

Sheldon M. , R. (2007). Introducción a la estdística segunda edición . Reverteré S.A.

Shigley, J., \& Mishchke, C. (2005). Diseño en Ingeniería Mecánica sexta edición. McGraw Hill.

Strozzi, A., Baldini , A., Giacopini, E., \& Mantovani , S. (2015). A repertoire of failures in connecting rods for internal combustion engines, and indications on traditional and advanced design methods. . Engineering failure analysis., 1-20.

Walpole, R. E. (1999). Probabilidad y Estadística para Ingenieros sexta edición . México: Prentice Hall Hispanamericana .

Westerman , G. (1986). Metalotecnia Fundamental . Barcelona : Reverteré . 